\title{
Nonparametric Estimation of Replacement Rates
}

\author{
Nora Dörmann
}

University of Duisburg-Essen, Germany

Corresponding Author: nora.doermann@uni-due.de

Copyright (c) 2017 by authors, all rights reserved. Authors agree that this article remains permanently open access under the terms of the Creative Commons Attribution License 4.0 International License

\begin{abstract}
Let $X_{i}, i \geq 1$, describe the lifetimes of items with finite mean $\mu=E\left(X_{i}\right)$ which are successively placed in service. In order to estimate the replacement rate $1 / \mu$ or related quantities, the random variables $X_{i}$ are usually assumed to be independent and identically distributed.

It is shown that a nonparametric estimation of the replacement rate and other reciprocal functions of renewal theory is possible while using a delta method with weakened requirements upon the global growth of $f$ which also allows dependent observations and respects the unboundedness of the analyzed reciprocal functions. Moreover, results on the moments and, furthermore, on corresponding simulations are included.
\end{abstract}

Keywords Rate of Replacement, Elementary Renewal Theorem, Delta Method, Weak Law of Large Numbers, Asymptotic Expansion of Moments, Dependent Observations

\section{Introduction}

Consider the problem of estimating the rate $1 / \mu$ of replacement based on a renewal process given by a sequence of random variables $X_{i}, i \geq 1$, representing lifetimes of items being renewed. The variables are assumed to be positive having the same mean $\mu$; they may be dependent and the common underlying distribution is unknown. As maximum likelihood methods are not applicable, the method of moments based upon substituting the $n$th sample mean $\bar{X}_{n}$ for $\mu$ is implemented, i.e., $1 / \mu$ is estimated by $1 / \bar{X}_{n}$. But this method is unfortunately not suitable for further derivation of corresponding moments of $1 / \mu$.

Based on the limiting moment approach, the so called delta method in its version for determination of moments and independent random variables is then preferred, cf. pp. 306 in Bickel and Doksum [1], pp. 429 in Lehmann and Casella [2], pp. 99 in Small [3] or pp. 25 in van der Vaart [4]. But, in either case, strict requirements on the boundedness of the function or its derivatives need to be fulfilled. As the rate $1 / \mu$ and its derivatives are unbounded - moreover, its graph decreases rapidly near the origin - the suggested methods in existing literature can consequently neither be chosen.

Meanwhile and in contrast to the literature, Weba and Dörmann [5] show that the well-known delta method is valid for a much broader class of functions. Provided mild requirements upon the global growth of $f$ are met, also functions where the function itself or all its derivatives are unbounded may be considered. Moreover, a quantitative version of the weak law of large numbers is sufficient and necessary for the approximation of $f$ and the expansion of its moments. Furthermore, observations are allowed to be dependent and no assumptions on their distributions are required.

Up to now, topics of renewal theory like the optimal adaptive replacement in a renewal process, see Robinson [6], or the prediction of failure, see Joshi [7], or nonparametric confidence intervals for the renewal function with censored data, see Baxter [8], are of main interest. The purpose of this paper is to apply this method on functions of renewal theory dependent on $\mu$ with dependent observations. As the analyzed functions are reciprocal, the unboundedness of the functions and its derivatives is respected by applying a quantitative version of the weak law of large numbers.

This paper is structured as follows: basic assumptions, notations and a brief outline on the application of the delta method to functions of the sample mean when observations are dependent are presented in section 2, see Weba and 
Dörmann [5]. Section 3 covers the application of this method on reciprocal functions. The paper closes in section 4 by giving numerical examples and illustrating the effectiveness of the proposed approach in simulations.

\section{The Delta Method for Dependent Observations}

In this chapter, basic assumptions and notations of the delta method for moments with dependent observations given by Weba and Dörmann [5] are summarized. Moreover, their formulated requirements and results on the expansions for bias and variance are shown. Besides, an alternative sufficient criterion replacing a quantitative version of the weak law of large numbers is presented as a closing remark.

\subsection{Basic Assumptions and Notations}

In regard of the renewal process, a sequence $X_{i}, i \geq 1$, of positive random variables is generally considered to represent the lifetimes of items being renewed, see p. 245 in Smith [9]. Additionally, each $X_{i}$ has finite mean $\mu=E\left(X_{i}\right)$, cf. p. 366 in Pinsky and Karlin [10].

All random variables take values in the open interval $I=(0, \infty)$. They are defined on a common probability space with underlying probability measure $P$. An indicator variable with respect to an event $B$ is denoted by $\mathbf{1}_{B}$.

As all variables $X_{i}$ have the same finite expectation $E\left(X_{i}\right)=\mu$, one has

$$
X_{i}=\mu+Y_{i}, \quad i \geq 1
$$

where $Y_{i}$ has expectation $E\left(Y_{i}\right)=0$.

For further analysis of a possible dependency structure, suppose that $p \geq 1$ is a fixed integer with $\left\|Y_{i}\right\|_{p}=\left(E\left(\left|Y_{i}\right|^{p}\right)\right)^{1 / p}<$ $\infty$ for all $i \geq 1$. The covariances of order $p$ are then given by

$$
E\left(Y_{i_{1}} \cdot Y_{i_{2}} \cdot \ldots \cdot Y_{i_{p}}\right), \quad i_{1} \geq 1, i_{2} \geq 1, \ldots, i_{p} \geq 1,
$$

and the quantity $S_{n, p}$ stands for

$$
S_{n, p}=\sum_{i_{1}=1}^{n} \sum_{i_{2}=1}^{n} \ldots \sum_{i_{p}=1}^{n} E\left(Y_{i_{1}} \cdot Y_{i_{2}} \cdot \ldots \cdot Y_{i_{p}}\right), \quad n \geq 1
$$

(where $S_{n, 1}=0$ ).

Moreover, the useful relation $\bar{X}_{n}=n^{-1} \cdot \sum_{i=1}^{n} X_{i}$

$$
E\left(\left(\bar{X}_{n}-\mu\right)^{p}\right)=n^{-p} \cdot S_{n, p}
$$

between $S_{n, p}$ and the arithmetic mean holds, and as $n \rightarrow \infty$ the behavior of $S_{n, p}$ depends upon the covariance structure. Furthermore, by the generalized Hoelder inequality, one also finds

$$
\left|S_{n, p}\right| \leq\left(\sum_{i=1}^{n}\left\|Y_{i}\right\|_{p}\right)^{p}
$$

\subsection{Expansions for Bias and Variance}

Consider the problems of estimating $f(\mu)$ by $f\left(\bar{X}_{n}\right)$ where $f: \mathbb{R} \rightarrow \mathbb{R}$ is a prescribed Borel measurable function and of deriving asymptotic expansions for bias and variance of $f\left(\bar{X}_{n}\right)$ as $n \rightarrow \infty$.

Therefore, assume conditions $(i),(i i)$ and (iii) on the differentiability of $f$ and on the asymptotic behavior of the quantity $S_{n, p}$ :

(i) $\quad f$ is $m \geq 1$ times continuously differentiable in a neighborhood of $\mu$.

(ii) As $n \rightarrow \infty$, the quantity $S_{n, p}$ satisfies $S_{n, p}=\mathcal{O}\left(n^{p}\right)$ for some even integer $p>m$.

(iii) $\quad f$ and $\bar{X}_{n}$ are related according to

$$
E\left(\left|f\left(\bar{X}_{n}\right)\right| \cdot \perp_{\left\{\left|\bar{X}_{n}-\mu\right| \geq \delta\right\}}\right)=\mathcal{O}\left(a_{n}\right) \text { for each } \delta>0
$$


where

$$
a_{n}=\left(\frac{S_{n, p}}{n^{p}}\right)^{\frac{m}{p}} .
$$

Here, condition (iii) expresses the weak law of large numbers in a quantitative version.

According to Theorem 1 in Weba and Dörmann [5], conditions (i), (ii) and (iii) imply expansion

$$
E\left(f\left(\bar{X}_{n}\right)\right)-f(\mu)=\sum_{j=2}^{m} \frac{f^{(j)}(\mu)}{j !} \cdot \frac{S_{n, j}}{n^{j}}+\mathcal{O}\left(a_{n}\right),
$$

whereas

$$
\lim _{n \rightarrow \infty} E\left(f\left(\bar{X}_{n}\right)\right)=f(\mu) .
$$

Likewise, the expansion for the variance follows if $\left(\right.$ iii) is replaced by $(i i i)^{\prime}$ :

$(\text { iii })^{\prime} \quad f$ and $\bar{X}_{n}$ are related according to

$$
E\left(f^{2}\left(\bar{X}_{n}\right) \cdot \mathbf{1}_{\left\{\left|\bar{X}_{n}-\mu\right| \geq \delta\right\}}\right)=\mathcal{O}\left(a_{n}\right)
$$

for each $\delta>0$.

Then, if $(i),(i i)$ and $(i i i)^{\prime}$ are satisfied, the variance admits representation

$$
\begin{aligned}
\operatorname{Var} & \left(f\left(\bar{X}_{n}\right)\right)= \\
& =\sum_{j=2}^{m}\left(\frac{\left(f^{2}\right)^{(j)}(\mu)-2 \cdot f^{(j)}(\mu) \cdot f(\mu)}{j !}\right) \cdot \frac{S_{n, j}}{n^{j}} \\
- & \sum_{(j, k) \in I_{m}} \frac{f^{(j)}(\mu)}{j !} \cdot \frac{f^{(k)}(\mu)}{k !} \cdot \frac{S_{n, j}}{n^{j}} \cdot \frac{S_{n, k}}{n^{k}}+\mathcal{O}\left(a_{n}\right)
\end{aligned}
$$

with index set $I_{m}=\{(j, k): j \geq 2, k \geq 2, j+k \leq m\}$. Especially,

$$
\lim _{n \rightarrow \infty} \operatorname{Var}\left(f\left(\bar{X}_{n}\right)\right)=0
$$

holds. (For the proof, see Theorem 1 of Weba and Dörmann [5].)

Expansions for the moments are provided by (7) and (9) in accordance with the particular conditions. Furthermore, Weba and Dörmann [5] also provide an alternative sufficient criterion for condition (iii) for a function $f$ which does not increase too rapidly "on the average". Results are also in practice for reciprocals and so applicated below.

For analysis of the global growth of $f$, suppose

$$
\left(E\left(\left|f\left(\bar{X}_{n}\right)\right|^{r}\right)\right)^{\frac{1}{r}} \leq c \cdot n^{s}, \quad n \geq 1
$$

to hold with constants $c>0, r>1$ and $s \geq 0$. Hence, conditions (11) and (12) turn out to be also sufficient for (5):

$$
p \cdot(r-1)>(m+s) \cdot r \quad \text { and } \quad S_{n, p}=\mathcal{O}\left(n^{\beta \cdot p}\right)
$$

where

$$
\beta=\frac{p \cdot(r-1)-(m+s) \cdot r}{p \cdot(r-1)-m \cdot r} .
$$

So, accuracy of $S_{n, p}=\mathcal{O}\left(n^{p}\right)$ is guaranteed by (11) and (12).

(For the proof, see Theorem 4 of Weba and Dörmann [5].)

As (11) with (12) is based on (10), the expectations $E\left(\left|f\left(\bar{X}_{n}\right)\right|^{r}\right)$ need further analysis. Therefore, it is more reasonable to inspect the expectations depending upon the single distributions of the random variables $X_{i}, \ldots, X_{n}$ rather 
than upon the distribution of their sum. Aside from this, it suffices to examine the asymptotic behavior of $E\left(f^{*}\left(\bar{X}_{n}\right)\right)$ as $n \rightarrow \infty$ when a convex function $f^{*}$ dominates $|f|^{r}$ on $I$.

So, suppose $f^{*}$ to be a convex function defined on $I$ which fulfills

$$
|f(t)|^{r} \leq f^{*}(t)
$$

for all $f \in I$ and

$$
\frac{1}{n} \cdot \sum_{i=1}^{n} E\left(f^{*}\left(X_{i}\right)\right) \leq C \cdot n^{\sigma}
$$

for all $n \geq 1$ with real constants $C>0, r>1$ and $\sigma \geq 0$.

Then the expansion

$$
\left(E\left(\left|f\left(\bar{X}_{n}\right)\right|^{r}\right)\right)^{\frac{1}{r}} \leq c \cdot n^{s}
$$

holds true with $c=C^{1 / r}$ and $s=\sigma / r$.

These results may be concluded out of

$$
\left|f\left(\bar{X}_{n}\right)\right|^{r} \leq f^{*}\left(\sum_{i=1}^{n} \frac{1}{n} \cdot X_{i}\right) \leq \sum_{i=1}^{n} \frac{1}{n} \cdot f^{*}\left(\bar{X}_{n}\right)
$$

which is especially applicable when $|f|^{r}$ itself is convex.

The existence of $E\left(X_{i}^{-r}\right)$ depends upon information about the behavior of $X_{i}$ near the origin. If $X_{i}$ follows a gamma distribution with parameters $\lambda_{i}>0, k_{i}>0$ - i.e., if $X_{i}$ has the density $h(x)=\lambda_{i}^{k_{i}} / \Gamma\left(k_{i}\right) \cdot x^{k_{i}-1} e^{-\lambda_{i} x}$ for $x>0$ (and $h(x)=0$ otherwise $)$ - then $E\left(X_{i}^{-r}\right)<\infty$ holds provided $r<k_{i}$.

\subsection{Estimation under Dependence}

As a detailed example based on a $\mathrm{MA}(q)$-process is presented in section 4, more explanation on the quantity $S_{n, p}$ representing a possible covariance structure of the underlying observations is needed. Therefore, the case $m=1$ and $p=2$ is discussed for weakly stationary processes. Its illustration can be carried over the example mentioned.

Then, suppose that $f$ is a prescribed function once continuously differentiable in a neighborhood of $\mu$ and the variables $X_{i}$ are assumed to have finite second moments; one consistently considers $m=1$ and $p=2$. In regard of the presented delta method in subsection 2.2 , one then needs to verify

$$
S_{n, 2}=\sum_{i=1}^{n} \sum_{j=1}^{n} E\left(Y_{i} Y_{j}\right)=\mathcal{O}\left(n^{2}\right)
$$

according to condition $(i i)$. Also, condition $(i i i)$ with

$$
E\left(\left|f\left(\bar{X}_{n}\right)\right| \cdot \mathbf{1}_{\left\{\left|\bar{X}_{n}-\mu\right| \geq \delta\right\}}\right)=\mathcal{O}\left(a_{n}\right) \quad \text { for each } \quad \delta>0
$$

where

$$
a_{n}=\frac{1}{n} \cdot \sqrt{S_{n, 2}}
$$

has to be checked.

Then representation in (7) results in

$$
E\left(f\left(\bar{X}_{n}\right)\right)-f(\mu)=\mathcal{O}\left(a_{n}\right)
$$

for the bias.

With

$$
E\left(f^{2}\left(\bar{X}_{n}\right) \cdot \mathbf{1}_{\left\{\left|\bar{X}_{n}-\mu\right| \geq \delta\right\}}\right)=\mathcal{O}\left(a_{n}\right)
$$


the variance out of (9) is given by

$$
\operatorname{Var}\left(f\left(\bar{X}_{n}\right)\right)=\mathcal{O}\left(a_{n}\right)
$$

In a next step, consider $Y_{i}, i \in \mathbb{Z}$ to be a weakly stationary time series. Its covariance function is given by $\gamma(h)=$ $E\left(Y_{i} Y_{i+h}\right)$. The representations for $S_{n, 2}$ and $a_{n}$ then become

$$
S_{n, 2}=\sum_{i=1}^{n} \sum_{j=1}^{n} \gamma(i-j)=\sum_{h=-(n-1)}^{n-1}(n-|h|) \cdot \gamma(h)
$$

and

$$
a_{n}=\frac{1}{n} \cdot \sqrt{\sum_{h=-(n-1)}^{n-1}(n-|h|) \cdot \gamma(h)}
$$

Then the estimate

$$
S_{n, 2} \leq n \cdot \sum_{h=-(n-1)}^{n-1}|\gamma(h)|=n^{2} \cdot\left(\frac{1}{n} \cdot \sum_{h=-(n-1)}^{n-1}|\gamma(h)|\right)
$$

is used for verification of $S_{n, 2}$.

As $\lim _{h \rightarrow \infty} \gamma(h)=0$, the expression $n^{-1} \cdot \sum_{h=-(n-1)}^{n-1}|\gamma(h)|$ also tends to 0 as $n \rightarrow \infty$ and one concludes $S_{n, 2}=\mathcal{O}\left(n^{2}\right)$.

\section{Reciprocal Functions in Renewal Theory}

The rate $1 / \mu$ of replacement is not only interesting in its own right; besides, it plays an important role in fundamental limit theorems of renewal theory. It is commonly known as elementary renewal theorem, see chapter 5 in Karlin and Taylor [11], section 5.6 in Lefebvre [12] or section 7.4.1 in Pinsky and Karlin [10].

Moreover, by way of comparison, application of the conventional delta method on this function where $X_{i}, i=1, \ldots, n$ are supposed to be independent, identically distributed and exponential random variables is given in example $4.33 \mathrm{on}$ pp. 208 in Knight [13].

Theorem 1 covers the application of the presented method on estimation of $1 / \mu$ by $1 / \bar{X}_{n}$. As the random variables $X_{i}$ are assumed to have finite fourth moments, $m=2$ and $p=4$ are here considered.

\section{Theorem 1}

Suppose conditions $(i),(i i)$ as in subsection 2.2 to be satisfied and assume additionally $S_{n, 4}=\mathcal{O}\left(n^{3}\right)$ and $n^{-1}$. $\sum_{i=1}^{n} E\left(X_{i}^{-4}\right)=\mathcal{O}(n)$.

Then the bias is given by

$$
E\left(\frac{1}{\bar{X}_{n}}\right)-\frac{1}{\mu}=\frac{1}{\mu^{3}} \cdot \frac{S_{n, 2}}{n^{2}}+\mathcal{O}\left(\frac{\sqrt{S_{n, 4}}}{n^{2}}\right)
$$

In particular, $\lim _{n \rightarrow \infty} E\left(\frac{1}{\bar{X}_{n}}\right)=\frac{1}{\mu}$ holds true.

For the variance, assume conditions $(i)$ and $(i i)$ and substitute $(i i i)^{\prime}$ by assumption of $S_{n, 4}=\mathcal{O}\left(n^{3}\right)$ and $n^{-1}$. $\sum_{i=1}^{n} E\left(X_{i}^{-8}\right)=\mathcal{O}(n)$.

Hence the variance admits representation

$$
\operatorname{Var}\left(\frac{1}{\bar{X}_{n}}\right)=\frac{1}{\mu^{4}} \cdot \frac{S_{n, 2}}{n^{2}}+\mathcal{O}\left(\frac{\sqrt{S_{n, 4}}}{n^{2}}\right)
$$

where $\lim _{n \rightarrow \infty} \operatorname{Var}\left(\frac{1}{\bar{X}_{n}}\right)=0$. 
Proof. Apparently, as the function $f$ is at least twice continuously differentiable in a neighborhood of $\mu$, condition $(i)$ is fulfilled and by $(3)$ condition $(i i)$ is also satisfied.

Analysis of condition ( iii) is realized by application of the alternative, sufficient result for the weak law of large numbers in (11) and (12). Therefore, by assumption of conditions $(i)$ and $(i i)$, consider additionally $S_{n, 4}=\mathcal{O}\left(n^{3}\right)$ as well as $n^{-1} \cdot \sum_{i=1}^{n} E\left(X_{i}^{-4}\right)=\mathcal{O}(n)$. Setting

$$
f(t)=\frac{1}{t}
$$

ensures convexity of $f^{4}(t)=t^{-4}$ on $I=(0, \infty)$. Application of the alternative criterion for the weak law of large numbers implies (10) with values $r=4, s=1 / 4$ for the constants.

For $m=2$, one may find $p \cdot(r-1)=12>(m+s) \cdot r=9$ as well as $\beta=3 / 4$ and, by assumption, $S_{n, 4}=$ $\mathcal{O}\left(n^{\beta \cdot 4}\right)=\mathcal{O}\left(n^{3}\right)$. Then (11) with (12) guarantees that $f$ and $\bar{X}_{n}$ are related according to (5). Consequently, the bias admits representation (13).

Moreover, imitating the arguments of the bias, conditions $S_{n, 4}=\mathcal{O}\left(n^{3}\right)$ and

$n^{-1} \cdot \sum_{i=1}^{n} E\left(X_{i}^{-8}\right)=\mathcal{O}(n)$ yield the result for the variance in $(14)$.

Allowing $X_{i}, i \geq 1$, to be continuous random variables with same finite mean $\mu$ and finite variance $\sigma^{2}$, the elementary renewal theorem may be extended to

$$
f\left(\mu, \sigma^{2}\right)=\frac{\sigma^{2}-\mu^{2}}{2 \mu^{2}},
$$

see p. 366-367 Pinsky and Karlin [10]. In this effect, it is reasonable to analyse the higher rate

$$
f(\mu)=\frac{1}{\mu^{2}}
$$

which is generalized to

$$
f(\mu)=\frac{1}{\mu^{\alpha}}
$$

with arbitrary $\alpha>0$. Furthermore, in the following, assumption of $m=2$ and $p=4$ is kept.

\section{Theorem 2}

Suppose that conditions $(i),($ ii $)$ and $($ iii $)$ hold whereas conditions $(i)$ and $(i i)$ may be transmitted as they are equal for the considered problem.

Condition (iii) is again replaced by considering $S_{n, 4}=\mathcal{O}\left(n^{3}\right)$ and $n^{-1} \cdot \sum_{i=1}^{n} E\left(\left(X_{i}\right)^{-4 \alpha}\right)=\mathcal{O}(n)$. Consequently, one obtains following representation regarding the bias:

$$
E\left(\frac{1}{\left(\bar{X}_{n}\right)^{\alpha}}\right)-\frac{1}{\mu^{\alpha}}=\frac{\alpha^{2}+\alpha}{2} \cdot \mu^{-(\alpha+2)} \cdot \frac{S_{n, 2}}{n^{2}}+\mathcal{O}\left(\frac{\sqrt{S_{n, 4}}}{n^{2}}\right)
$$

which entails that $1 /\left(\bar{X}_{n}\right)^{\alpha}$ is asymptotically unbiased for $1 / \mu^{\alpha}$ as $n \rightarrow \infty$.

Furthermore, in regard of the variance, assume conditions $(i),($ ii $)$ and $(i i i)^{\prime}$ whereas $(i i i)^{\prime}$ is substituted by assumption of $S_{n, 4}=\mathcal{O}\left(n^{3}\right)$ and $n^{-1} \cdot \sum_{i=1}^{n} E\left(\left(X_{i}\right)^{-8 \alpha}\right)=\mathcal{O}(n)$.

Then the variance admits representation

$$
\operatorname{Var}\left(\frac{1}{\left(\bar{X}_{n}\right)^{\alpha}}\right)=\alpha^{2} \cdot \mu^{-(2 \alpha+2)} \cdot \frac{S_{n, 2}}{n^{2}}+\mathcal{O}\left(\frac{\sqrt{S_{n, 4}}}{n^{2}}\right)
$$

where $\lim _{n \rightarrow \infty} \operatorname{Var}\left(1 /\left(\bar{X}_{n}\right)^{\alpha}\right)=0$.

Proof. With $m=2$ and $p=4$, condition $(i)$ certainly holds true for the considered function $f$ as well as condition $(i i)$ by $(3)$. 
As already stated, accuracy of condition (iii) is also ensured by the alternative sufficient criterion which is used for analysis of

$$
f(t)=\frac{1}{t^{\alpha}}
$$

where $t>0$ and for arbitrary $\alpha>0$.

Consequently, as $n \rightarrow \infty$, the asymptotic behavior of $E\left(f^{*}\left(\bar{X}_{n}\right)\right)$ needs to be examined where $f^{*}$ is supposed to be a convex function which dominates $|f|^{r}$ on $I$. Therefor, consider $r=4$ and assume $f^{*}$ to be a convex function defined on $I$ which fulfills

$$
|f(t)|^{4}=\frac{1}{t^{4 \alpha}}
$$

for all $f \in I$ and

$$
\frac{1}{n} \cdot \sum_{i=1}^{n} E\left(f^{*}\left(X_{i}\right)\right) \leq C \cdot n^{\sigma}
$$

for all $n \geq 1$ with real constants $C>0, r>1$ and $\sigma \geq 0$.

Then, as $S_{n, 4}=\mathcal{O}\left(n^{3}\right)$ and

$$
\frac{1}{n} \cdot \sum_{i=1}^{n} E\left(f^{*}\left(X_{i}\right)\right)=\frac{1}{n} \cdot \sum_{i=1}^{n} E\left(\frac{1}{\left(X_{i}\right)^{4 \alpha}}\right)=\mathcal{O}(n)
$$

are supposed, convexity of $f^{4}(t)=t^{-4 \alpha}$ is guaranteed on $I=(0, \infty)$. The application of this alternative criterion for the weak law of large numbers implies (10) with values $r=4, \sigma=1$ and $s=1 / 4$.

Consequently, by $m=2$, one may find $p \cdot(r-1)=12>(m+s)=9$ and besides $\beta=3 / 4$. Relation of $f$ and $\bar{X}_{n}$ given in (5) is then guaranteed by (11) with (12).

By (7) and assuming $(i),(i i)$ and (iii), the bias admits representation in (17) which entails asymptotic unbiasedness.

Computation of the variance relies on imitating the arguments of the bias and assumption of condition $S_{n, 4}=\mathcal{O}\left(n^{3}\right)$ and $n^{-1} \cdot \sum_{i=i}^{n} E\left(\left(X_{i}\right)^{-8 \alpha}\right)=\mathcal{O}(n)$. The expansion for the variance is then given by (18).

\section{Applications and Simulations}

By an example this chapter illustrates results on estimation of reciprocal functions $f(\mu)$ of renewal theory by $f\left(\bar{X}_{n}\right)$ and deriving asymptotic expansions for its moments by using the presented delta method. In addition to the results of the analyzed functions, information on the arithmetic mean and the quantity $S_{n, p}$ are stated according to the selected sample size $n$ when necessity is considered needed.

\subsection{Setting and Inspection of Requirements}

Suppose one wants to estimate the rate $1 / \mu$ of replacement whereas the values of the variables $X_{i}$ result from a $\operatorname{MA}(q)$ process.

Therefore, assume at first $\xi_{j}, j \geq 0$, to be a sequence of independent and identically distributed random variables obeying a gamma-distribution $\xi_{j} \sim \Gamma(\lambda, k)$ with $\lambda, k>0$ and corresponding density. (This distribution is chosen as a general case of the exponentional distribution which is commonly used for examples with positive random variables in renewal theory.) Then the variables $X_{i}$ describe a MA(q)-process given by $X_{i}=\sum_{j=0}^{q} \theta_{j} \cdot \xi_{i-j}$ with $q \geq 0$ and $\theta_{j}>0$. So one finds

$$
E\left(X_{i}\right)=\left(\sum_{j=0}^{q} \theta_{j}\right) \cdot \frac{k}{\lambda}=: \mu
$$


for each $X_{i}$ and consequently, representation in (1) also holds in this case as

$$
\begin{aligned}
X_{i} & =\sum_{j=0}^{q} \theta_{j} \cdot\left(\xi_{i-j}-E\left(\xi_{i-j}\right)\right)+\sum_{j=0}^{q} \theta_{j} \cdot E\left(\xi_{i-j}\right) \\
& =\sum_{j=0}^{q} \theta_{j} \cdot Z_{i-j}+\left(\sum_{j=0}^{q} \theta_{j}\right) \cdot \frac{k}{\lambda} \\
& =: \quad Y_{i}+\mu
\end{aligned}
$$

where $Y_{i}=\sum_{j=0}^{q} \theta_{j} \cdot Z_{i-j}$. Moreover, $E\left(Z_{j}\right)=0$ and $\operatorname{Var}\left(Z_{j}\right)=k / \lambda^{2}$ are assumed.

Being a moving average process of order $q, Y_{i}$ is weakly stationary with $E\left(Y_{i}\right)=0$ and covariance structure

$$
\gamma(h)=\left\{\begin{array}{ll}
0, & |h|>q \\
\frac{k}{\lambda^{2}} \cdot \sum_{i=0}^{q-|h|} \theta_{i} \theta_{i+|h|}, & |h| \leq q
\end{array} .\right.
$$

Moreover, the required quantity $S_{n, 2}$ may be estimated by

$$
S_{n, 2}=\sum_{h=-(n-1)}^{n-1}(n-|h|) \cdot \gamma(h) \leq n \cdot \sum_{h=-(n-1)}^{n-1}|\gamma(h)|=\mathcal{O}(n)
$$

As the regarded function is evidently at least twice continuously differentiable in a neighborhood of $\mu$, condition $(i)$ holds. By (3) condition $(i i)$ is met. Condition $($ iii $)$ is replaced by the alternative requirements.

(i) Requirements for the bias: Provided relations $S_{n, 4}=\mathcal{O}\left(n^{3}\right)$ and $n^{-1} \cdot \sum_{i=1}^{n} E\left(X_{i}^{-4}\right)=\mathcal{O}(n)$ hold, the bias is given by $(13)$.

In fact, even $S_{n, 4}=\mathcal{O}\left(n^{2}\right)$ holds in case of $\sup _{i \in \mathbb{Z}} E\left(Z_{i}^{4}\right)<\infty$, see 4.3 (iii) in Weba and Dörmann [5], which applies as $Z_{i}=\xi_{i}-\lambda / k$ and as $E\left(\xi_{i}\right)=k / \lambda, E\left(\xi_{i}^{2}\right)<\infty, E\left(\xi_{i}^{3}\right)<\infty$ and $E\left(\xi_{i}^{4}\right)<\infty$ are independent of $i$ respectively.

The second requirement is fulfilled, e.g., for $(q+1) \cdot k>4$. Then the estimate

$$
X_{i}=\sum_{j=0}^{q} \theta_{j} \cdot \xi_{i-j} \geq\left(\min _{j=0, \ldots, q} \theta_{j}\right) \cdot \sum_{j=0}^{q} \xi_{i-j}
$$

becomes

$$
X_{i}=\sum_{j=0}^{q} \theta_{j} \cdot \xi_{i-j} \geq \hat{\theta} \cdot \hat{\xi}_{i}
$$

with $\hat{\theta}=\left(\begin{array}{c}\min \theta_{j} \\ j=0, \ldots, q\end{array}\right), \hat{\xi}_{i}=\sum_{j=0}^{q} \xi_{i-j}, \hat{\theta}>0 . \hat{\xi}_{i} \sim \Gamma(\lambda,(q+1) \cdot k)$ is used for analysis of

$$
\frac{1}{X_{i}^{4}} \leq\left(\frac{1}{\hat{\theta}}\right)^{4} \cdot \hat{\xi}_{i}^{-4}
$$

Taking expectations on both sides leads to

$$
E\left(\frac{1}{X_{i}^{4}}\right) \leq \hat{\theta}^{-4} \cdot E\left(\hat{\xi}_{i}^{-4}\right)
$$

with

$$
E\left(\hat{\xi}_{i}^{-4}\right)=\frac{\Gamma((q+1) \cdot k-4)}{\Gamma((q+1) \cdot k) \cdot \lambda^{-4}}<\infty
$$

as $-4>-(q+1) \cdot k$ and $4<(q+1) \cdot k$ respectively. This actually implies $n^{-1} \cdot \sum_{i=1}^{n} E\left(X_{i}^{-4}\right)=\mathcal{O}(1)$. 
(ii) Requirements for the variance: Meeting $S_{n, 4}=\mathcal{O}\left(n^{3}\right)$ and $n^{-1} \cdot \sum_{i=1}^{n} E\left(X_{i}^{-8}\right)=\mathcal{O}(n)$, representation in (14) holds for the variance. For the quantity $S_{n, 4}$, the above argumentation carries over. The second requirement is verified analogously to $(i)$ in view of

$$
\frac{1}{X_{i}^{8}} \leq\left(\frac{1}{\hat{\theta}}\right)^{8} \cdot \hat{\xi}_{i}^{-8}
$$

Again, taking expectations on both sides gives

$$
E\left(\frac{1}{X_{i}^{8}}\right) \leq \hat{\theta}^{-8} \cdot E\left(\hat{\xi}_{i}^{-8}\right)
$$

with

$$
E\left(\hat{\xi}_{i}^{-8}\right)=\left(\frac{\Gamma(q+1) \cdot k-8}{\Gamma((q+1) \cdot k) \cdot \lambda^{-8}}\right)<\infty
$$

for $-8>-(q+1) \cdot k$ or $8<(q+1) \cdot k$ respectively.

\subsection{Results of Simulation}

For estimation of $1 / \mu$ by $1 / \bar{X}_{n}$ and deriving asymptotic moments, suppose $\xi_{i}$ to be independent and identically distributed random variables with $\xi_{i} \sim \Gamma(10,10)$ and $E\left(\xi_{i}\right)=1$. Furthermore, consider $X_{i}$ to be a MA(4)-process having the representation

$$
X_{i}=0.08 \cdot \xi_{i}-0.005 \cdot \xi_{i-1}+0.01 \cdot \xi_{i-2}-0.005 \cdot \xi_{i-3}+0.02 \cdot \xi_{i-4} .
$$

with $E\left(X_{i}\right)=\mu=0.1$.

In a next step, under consideration of an increasing sample sizes $n$ and $\mu=0.1$, intermediate findings on the sample means $\bar{X}_{n}$, the reciprocals $1 / \bar{X}_{n}$ and the quantity $S_{n, 2}$ for $X_{i}$ are given in table 1 .

Table 1. Intermediate findings.

\begin{tabular}{cccc}
\hline$n$ & $\bar{X}_{n}$ & $\bar{X}_{n}^{-1}$ & $S_{n, 2}$ \\
\hline 50 & 0.11544279 & 8.66229916 & 0.59619955 \\
100 & 0.10886109 & 9.18601823 & 0.78518996 \\
1000 & 0.10080687 & 9.91995785 & 0.65105512 \\
5000 & 0.09966694 & 10.03341657 & 2.77310442 \\
10000 & 0.09997542 & 10.00245835 & 0.06040523 \\
50000 & 0.10004456 & 9.99554583 & 4.96430860 \\
75000 & 0.10012190 & 9.98782384 & 83.59898518 \\
100000 & 0.09997542 & 10.00245835 & 78.98798390 \\
\hline
\end{tabular}

Furthermore, corresponding results of computation for the estimated bias and the estimated variance are given in table 2. Representations in (13) and (14) have been used without the unknown error terms. Also empirical results for the considered moments are included. For that purpose, simulation was repeated 50 times. For each of them, the particular mean was calculated. Then an empirical value for their expectation was consequently deduced by averaging the mentioned means and considered for the deviation of the bias. Likewise, the value of the empirical variance is also computed.

Table 2. Results of simulation.

\begin{tabular}{ccccc}
\hline$n$ & estimated bias & empirical bias & estimated variance & empirical variance \\
\hline 50 & 0.23847982 & 0.02467609 & 2.38479820 & 0.30746232 \\
100 & 0.07851899 & -0.01224402 & 0.78518996 & 0.12003545 \\
1000 & 0.00065105 & -0.00268349 & 0.00651055 & 0.01564937 \\
5000 & 0.00011092 & 0.00197195 & 0.00110924 & 0.00169001 \\
10000 & 0.00000060 & -0.00062126 & 0.00000604 & 0.00084053 \\
50000 & 0.00000198 & -0.00104865 & 0.00001985 & 0.00014872 \\
75000 & 0.00001486 & -0.00031878 & 0.00014862 & 0.00011649 \\
100000 & 0.00000789 & 0.00016548 & 0.00007898 & 0.00008487 \\
\hline
\end{tabular}


Results of simulation show asymptotic unbiasedness and consistency as well as the rate of convergence.

Assuming alternative values for the parameters $k$ and $\lambda$ gives following results on the moments and the rate of convergence. Therefore, two cases are described in table 3 and 4 .

Table 3. Results of simulation with diverse values of parameters $\xi_{i} \sim \Gamma(200,1000)$.

\begin{tabular}{ccc}
\hline & \multicolumn{2}{c}{$\xi_{i} \sim \Gamma(200,1000)$} \\
$n$ & estimated bias & estimated variance \\
\hline 50 & 0.0003039746 & 0.0006079493 \\
100 & 0.0001287686 & 0.0002575373 \\
1000 & 0.0000006248 & 0.0000012497 \\
5000 & 0.0000001172 & 0.0000002344 \\
10000 & 0.0000000003 & 0.0000000007 \\
50000 & 0.0000000073 & 0.0000000147 \\
75000 & 0.0000000263 & 0.0000000526 \\
100000 & 0.0000000157 & 0.0000000314
\end{tabular}

Table 4. Results of simulation with diverse values of parameters $\xi_{i} \sim \Gamma(500,100)$.

\begin{tabular}{ccc}
\hline & \multicolumn{2}{c}{$\xi_{i} \sim \Gamma(500,100)$} \\
$n$ & estimated bias & estimated variance \\
\hline 50 & 0.07920846 & 3.96042320 \\
100 & 0.03298830 & 1.64941529 \\
1000 & 0.00014681 & 0.00734067 \\
5000 & 0.00003704 & 0.00185223 \\
10000 & 0.00000005 & 0.00000275 \\
50000 & 0.00000151 & 0.00007580 \\
75000 & 0.00000698 & 0.00034914 \\
100000 & 0.00000401 & 0.00020094 \\
\hline
\end{tabular}

\subsection{Generalizations}

As shown in Theorem 2, the more general results for estimation of $f(\mu)=1 / \mu^{\alpha}$ by $f\left(\bar{X}_{n}\right)=1 /\left(\bar{X}_{n}\right)^{\alpha}$ in $(17)$ and (18) hold as well. Their illustration by simulation in context of the established setting completes this chapter. Therefor, the needed conditions and requirements are again checked and results of simulation provided.

\subsubsection{Inspection of Requirements}

With $m=2$ and $p=4$, condition $(i)$ is ensured as the considered function is at least twice continuously differentiable in a neighborhood of $\mu$. By (3), condition (ii) holds also in this case. Condition (iii) is replaced by the shown alternative requirements for both moments.

(i) Requirements for the bias: Again $S_{n, 4}=\mathcal{O}\left(n^{3}\right)$ needs to be fulfilled. For its argumentation one may refer to $4.1(i)$.

Motivation of the second requirement $n^{-1} \cdot \sum_{i=1}^{n} E\left(\left(X_{i}\right)^{-4 \alpha}\right)=\mathcal{O}(n)$ is given analogously to $4.1(i)$. Therefor, assume again $\hat{\xi}_{i} \sim \Gamma(\lambda,(q+1) \cdot k)$ and proceed with inspection of the estimate

$$
\frac{1}{X_{i}^{4 \alpha}} \leq\left(\frac{1}{\hat{\theta}}\right)^{4 \alpha} \cdot \hat{\xi}_{i}^{-4 \alpha} .
$$

Taking expectations yields

$$
E\left(\frac{1}{X_{i}^{4 \alpha}}\right) \leq \hat{\theta}^{-4 \alpha} \cdot E\left(\hat{\xi}_{i}^{-4 \alpha}\right)
$$


with

$$
E\left(\hat{\xi}_{i}^{-4 \alpha}\right)=\frac{\Gamma((q+1) \cdot k-4 \alpha)}{\Gamma((q+1) \cdot k) \cdot \lambda^{-4 \alpha}} \leq \infty
$$

in case of $-4 \alpha>-(q+1) \cdot k$ and $4 \alpha<(q+1) \cdot k$ respectively. Again, $n^{-1} \cdot \sum_{i=1}^{n} E\left(X_{i}^{-4 \alpha}\right)=\mathcal{O}(1)$ also holds.

(i) Requirements for the variance: In regard of the variance, not only $S_{n, 4}=\mathcal{O}\left(n^{3}\right)$ but also $n^{-1} \cdot \sum_{i=1}^{n} E\left(X_{i}^{-8 \alpha}\right)=\mathcal{O}(n)$ are required. As to the quantity $S_{n, 4}$, see $4.1(i)$. For the second requirement, consider again $\hat{\xi}_{i} \sim \Gamma(\lambda,(q+1) \cdot k)$ and continue analogously with

$$
\frac{1}{X_{i}^{8 \alpha}} \leq\left(\frac{1}{\hat{\theta}}\right)^{8 \alpha} \cdot \hat{\xi}_{i}^{-8 \alpha}
$$

Taking expectations leads to

$$
E\left(\frac{1}{X_{i}^{8 \alpha}}\right) \leq \hat{\theta}^{-8 \alpha} \cdot E\left(\hat{\xi}_{i}^{-8 \alpha}\right)
$$

with

$$
E\left(\hat{\xi}_{i}^{-8 \alpha}\right)=\frac{\Gamma((q+1) \cdot k-8 \alpha)}{\Gamma((q+1) \cdot k) \cdot \lambda^{-8 \alpha}} \leq \infty
$$

for $-8 \alpha>-(q+1) \cdot k$ and $8 \alpha<(q+1) \cdot k$ respectively. Again, $n^{-1} \cdot \sum_{i=1}^{n} E\left(X_{i}^{-8 \alpha}\right)=\mathcal{O}(1)$ also holds.

\subsubsection{Results of Simulation}

Completion of examples is done by applying the already given setting in 4.2 with (17) and (18) for an arbitrary chosen exponent $\alpha>0$ whereas again only the approximation terms are considered and the unknown error term again neglected. Therefore assume $\xi_{i} \sim \Gamma(10,10)$ with $E\left(\xi_{i}\right)=1$ and consider for $X_{i}$ again the described MA(4)-process. Table 5 shows results; thereby intermediate findings are omitted. Empirical values of the moments are analogously computed as in subsection 4.2 . 
Table 5. Results of simulation with arbitrary exponent $\alpha>0$.

\begin{tabular}{|c|c|c|c|c|}
\hline \multicolumn{5}{|c|}{$\alpha=0.5$} \\
\hline$n$ & estimated bias & empirical bias & estimated variance & empirical variance \\
\hline 50 & 0.02828022 & 0.00389922 & 0.05961995 & 0.00761365 \\
\hline 100 & 0.00931120 & -0.00193654 & 0.01962974 & 0.00298361 \\
\hline 1000 & 0.00007720 & -0.00042432 & 0.00016276 & 0.00039083 \\
\hline 5000 & 0.00001315 & 0.00031177 & 0.00002773 & 0.00004226 \\
\hline 10000 & 0.00000007 & -0.00009823 & 0.00000015 & 0.00002102 \\
\hline 50000 & 0.00000023 & -0.00016581 & 0.00000049 & 0.00000371 \\
\hline 75000 & 0.00000176 & -0.00005040 & 0.00000371 & 0.00000291 \\
\hline 100000 & 0.00000093 & 0.00002616 & 0.00000197 & 0.00000212 \\
\hline \multicolumn{5}{|c|}{$\alpha=1.5$} \\
\hline$n$ & estimated bias & empirical bias & estimated variance & empirical variance \\
\hline 50 & 1.41401139 & 0.11712117 & 53.65795959 & 7.00268387 \\
\hline 100 & 0.46556038 & -0.05806070 & 17.66677428 & 2.72022835 \\
\hline 1000 & 0.00386028 & -0.01272809 & 0.14648740 & 0.35251477 \\
\hline 5000 & 0.00065769 & 0.00935428 & 0.02495793 & 0.03801616 \\
\hline 10000 & 0.00000358 & -0.00294685 & 0.00013591 & 0.01890513 \\
\hline 50000 & 0.00001177 & -0.00497408 & 0.00044678 & 0.00334607 \\
\hline 75000 & 0.00008812 & -0.00151213 & 0.00334395 & 0.00262126 \\
\hline 100000 & 0.00004683 & 0.00078496 & 0.00177722 & 0.00190991 \\
\hline \multicolumn{5}{|c|}{$\alpha=3$} \\
\hline$n$ & $\begin{array}{l}\text { estimated bias } \\
(1.0 \mathrm{e}+02 \cdot)\end{array}$ & $\begin{array}{c}\text { empirical bias } \\
(1.0 \mathrm{e}+02 \cdot)\end{array}$ & $\begin{array}{l}\text { estimated variance } \\
\qquad(1.0 \mathrm{e}+05 \cdot)\end{array}$ & $\begin{array}{c}\text { empirical variance } \\
(1.0 \mathrm{e}+05 \cdot)\end{array}$ \\
\hline 50 & 1.43087892 & 0.07421110 & 2.14631838 & 0.29516679 \\
\hline 100 & 0.47111398 & -0.03668710 & 0.70667097 & 0.11212230 \\
\hline 1000 & 0.00390633 & -0.00804833 & 0.00585949 & 0.01415881 \\
\hline 5000 & 0.00066554 & 0.00591704 & 0.00099831 & 0.00151961 \\
\hline 10000 & 0.00000362 & -0.00186366 & 0.00000543 & 0.00075541 \\
\hline 50000 & 0.00001191 & -0.00314563 & 0.00001787 & 0.00013381 \\
\hline 75000 & 0.00008917 & -0.00095633 & 0.00013375 & 0.00010487 \\
\hline 100000 & 0.00004739 & 0.00049646 & 0.00007108 & 0.00007641 \\
\hline
\end{tabular}

Out of the results, the rate of convergence is for direct reading. As it is for $\alpha=3$ significantly slower than for $\alpha=1.5$, it is faster in comparison with the other values when $\alpha=0.5$.

\section{REFERENCES}

[1] P. J. Bickel, K. A. Doksum. Mathematical Statistics - Basic Ideas and Selected Topics, Prentice Hall, 2001.

[2] E. L. Lehmann, G. Casella. Theory of Point Estimation, Springer, 2003.

[3] C. G. Small. Expansions and Asymptotics for Statistics, CRC Press, 2010.

[4] A. W. van der Vaart. Asymptotic Statistics, Cambridge University Press, 2000.

[5] M. Weba, N. Dörmann. Application of the delta method to functions of the sample mean when observations are dependent, Statistical Papers (Published; doi:10.1007/s00362-015-0734-7), 2015. 
[6] N. Robinson, K. Aboura. Optimal adaptive replacement in a renewal process, Applied Stochastic Models in Business and Industry, Vol. 31, 424-434, 2015

[7] B. H. Joshi. Smith. Prediction of failure - a case study, Journal of Applied Statistics, Vol. 22, No. 4, 458-493, 1995.

[8] L. A. Baxter, L. Li. Nonparametric confidence intervals for the renewal function with censored data, Journal of Nonparametric Statistics, Vol. 4, 317-326, 1995.

[9] W. L. Smith. Renewal theory and its ramifications, Journal of the Royal Statistical Society, Series B (Methodological), Vol. 20, No. 2, 243-302, 1958.

[10] M. A. Pinsky, S. Karlin. An Introduction to Stochastic Modeling, Academic Press, 2011.

[11] S. Karlin, H. M. Taylor. A First Course in Stochastic Processes, Academic Press, 1975.

[12] M. Lefebvre. Applied Stochastic Processes, Springer, 2007.

[13] K. Knight. Mathematical Statistics, Chapman \& Hall/CRC, 2000. 